\title{
Diferenciais de salários por gênero na Indústria Avícola da Região Sul do Brasil: uma análise com micro dados*
}

Paulo de Andrade Jacinto**

Resumo: Este trabalho analisa os diferenciais de salários por gênero na indústria avícola da Região Sul do Brasil, tendo como base as informações da Relação Anual de Informações Sociais (RAIS) de 1998. Para tanto, a decomposição de Oaxaca (1973) é empregada para mensurar as diferenças de salários entre trabalhadores homogêneos quanto à educação e ocupação. Os resultados do trabalho mostram que a discriminação que ocorre no mercado de trabalho como um todo também está presente nessa indústria, ou seja, encontraram-se evidências da existência de diferenciais de salários favoráveis aos homens.

Palavras chave: diferencial de salários, decomposição de Oaxaca, gênero.

Classificação JEL: J71, D82.

Abstract: This paper examines the wage differentials by gender in the poultry industry from South Region of Brazil. The Relação Anual de Informações Sociais - RAIS of 1998 was used. To measure the difference between the homogeneous workers as education and occupation it was used the

* O Autor agradece aos referees pelos comentários e sugestões, reconhecendo a responsabilidade pelos eventuais erros remanescentes.

** Doutorando do Programa de Pós-Graduação em Economia - PPGE/UFRGS e Professor da Universidade de Passo Fundo. paj@ppge.ufrgs.brou paulo@upf.br 
Oaxaca's method (1973). The results showed that the same discrimination found in the labor markets is present into the poultry industry.

Key words: wage differentials, Oaxaca decomposition, gender.

JEL Classification: J71, D82.

\section{1- Introdução}

O sucessivo aumento da participação feminina no mercado de trabalho pode ser considerado um dos mais importantes fatos observados nos últimos anos. A redução dos postos de trabalho na indústria e o aumento do desemprego e da informalidade também foram marcantes, porém nada se compara com o crescimento da força de mão-de-obra feminina. Para se ter uma idéia da intensidade desse aumento, segundo dados do IBGE, em 1970, a taxa de participação das mulheres na população economicamente ativa (PEA) correspondia a 20,86\%. Em 1990, essa taxa atingia $35,56 \%$, ou seja, em menos de vinte anos a participação feminina quase dobrou. As razões que levaram a esse crescimento não vêm ao caso nesse momento, porém o fato é que os números impressionam e têm desencadeado uma série de estudos, entre os quais se destacam os de Barros e Mendonça (1989), Jatobá (1990), Ramos e Soares (1995), Ometto (1997), Ometto, Hoffmann e Alves (1999), Scorzafave e Menezes-Filho (2001) e Fernandes e De Felício (2002).

Embora esse crescimento possa ser visto como uma transformação positiva que vem ocorrendo no mercado de trabalho, as implicações que decorrem dele têm sido marcadas por um fenômeno conhecido como discriminação salarial do trabalho feminino. Essa discriminação surge quando trabalhadores cuja produtividade é considerada idêntica recebem salários diferentes, tendo como base algumas características que não são consideradas produtivas ${ }^{1}$, ou porque o mercado de trabalho é segmentado. Segundo Ometto, Hoffmann e Alves (1999), até o ano de 1985 o rendimento real das mulheres manteve-se em torno de $50 \%$ daquele dos homens. Porém, a partir daí, essa remuneração cresceu

\footnotetext{
${ }^{1}$ Existência de diferenciais de salários entre grupo de trabalhadores após controlar por diferenças em atributos como educação, idade e características dos postos de trabalho.
} 
proporcionalmente mais do que a masculina, em 1986, ou caiu menos, em 1987, 1988 e 1990, de modo que, ao final do período, a remuneração das mulheres passou a representar 57,6\% da dos homens.

A principal razão para o aumento do rendimento médio mensal está associada ao papel da mulher na sociedade e, por conseqüência, a sua maior inserção no mercado de trabalho. Isso demonstra que deixou de ser apenas um trabalho visando à complementação de renda e passa a ser uma questão de necessidade econômica. Segundo Sanches (2000) a proporção de mulheres como chefe de família no Brasil aumentou 5,6 pontos percentuais em apenas oito anos. Entretanto, a existência de um diferencial de salários associado ao período de tempo necessário para que ela encontre emprego pode inibir a participação das mulheres no mercado de trabalho.

Na literatura econômica, uma série de trabalhos de natureza empírica, como o de Macedo (1985), Barros, Ponte e Varandas (1988), Reis e Barros (1991), Azevedo (1997), Ometto (1997), Carrera-Fernandez e Menezes (1998), Cavalieri e Fernandes (1998), Ometto, Hoffmann e Alves (1999), Jansen (1999), Sanches (2000) e Ikeda (2000), utilizando informações disponibilizadas pela Pesquisa Nacional de Amostra e Domicílios (PNAD) ou pela Pesquisa de Emprego e Desemprego (PED), indicou a existência de diferenciais de salários entre os trabalhadores homogêneos com respeito à sua qualificação, mas lotados em postos de trabalho distintos. Em menor quantidade, esse mesmo resultado foi observado para discriminação por gênero.

Tal fato, no entanto, encobriu algumas especificidades apresentadas nos diversos setores econômicos, como no caso da indústria avícola. Nessa indústria, talvez essa situação possa ser revertida, pois as empresas, ao anunciarem vagas para o setor de produção, buscam preferencialmente contratar trabalhadores do sexo feminino em razão de suas habilidades manuais, como, por exemplo, no abate, no corte e na limpeza das aves.

Nesse caso, os fatores considerados como inibidores da participação da mulher no mercado de trabalho, bem como determinaram a existência de um diferencial de salário em favor dos homens, podem reverter a favor das mulheres, o que pode determinar, portanto, uma redução do referido diferencial ou, até mesmo, a sua reversão. Dessa forma, o objetivo do presente artigo é identificar as diferenças salariais no setor 
de carnes, mais especificamente, na indústria avícola da região Sul do Brasil, para o ano de 1998, empregando a decomposição de Oaxaca para responder às questões como: existem diferenças salariais por gênero? Caso existam, quais são os níveis de diferenças salariais?

A despeito dos estudos realizados no Brasil, a contribuição deste estudo para a literatura pode ser vista em duas direções: a primeira atende à necessidade de verificar até que ponto a discriminação por gênero está presente ao se controlar pela educação e para uma ocupação específica dentro dessa indústria, qual seja, magarefe (sinônimo de retalhador, açougueiro e abatedor); a segunda, no uso das informações das características individuais, como educação, idade, experiência obtida na Relação Anual de Informações Sociais - RAIS. Assim, além dessa breve introdução, a segunda seção descreve de forma sucinta a economia da discriminação, a terceira apresenta a decomposição de Oaxaca, a quarta traz os resultados encontrados e, por fim, a seção cinco apresenta as conclusões.

\section{2- Economia da discriminação}

Antes de descrever como mensurar a discriminação, é importante apresentar como ela vem sendo tratada pelos economistas ${ }^{2}$. Em termos literais, a discriminação pode ser vista como diferenciação ou distinção entre objetos ou indivíduos que, ao ser estendida à economia, adquire uma conotação pejorativa. Por exemplo, a existência de diferenças de ganhos e de oportunidades de emprego entre trabalhadores com habilidades iguais no mesmo emprego. Trata-se de um termo que é, segundo Oaxaca (1987), geralmente empregado para distinções que são socialmente inaceitáveis e economicamente ineficientes. Considerando o exemplo acima, a ineficiência é no sentido de alocação de recursos na qual os indivíduos não são eficientemente alocados no mercado de trabalho em virtude da discriminação, vindo a resultar em salários desiguais, em tratamento diferenciado, ou mesmo em segregação.

\footnotetext{
${ }^{2}$ Uma leitura mais detalhada sobre o tema pode ser feita em Loureiro (2003) cujo trabalho faz uma resenha teórica e empírica sobre a economia da discriminação; em Arbache (2000), que apresenta um estudo sobre a determinação e diferencial de salários no Brasil, e em Cain (1986), que faz uma análise econômica da discriminação no mercado de trabalho.
} 
Com relação ao tipo de discriminação encontrada no mercado de trabalho, segundo Loureiro (2003), pode ser considerada como discriminação salarial, de emprego, de trabalho ou ocupacional ou, ainda, discriminação ao acesso de capital humano. É mais comum observar os três primeiros tipos após os indivíduos se encontrarem alocados, isto é, uma discriminação pós-mercado, ao passo que o último se verifica antes do indivíduo entrar no mercado de trabalho, ou seja, uma discriminação pré-mercado.

Os modelos elaborados pelos economistas visando entender melhor esse problema e suas conseqüências para a economia compõem a teoria econômica da discriminação e podem ser classificados em três categorias. A primeira está relacionada ao poder econômico no qual o uso do poder de monopólio dá origem à discriminação. Entre os colaboradores dessa categoria, segundo Oaxaca (1987), encontram-se Robinson (1933), Bergmann (1971) e Madden (1975). Para eles, a origem da discriminação pode ser compreendida supondo-se a existência de empregador monopsonista e que os trabalhadores homens e mulheres são substitutos perfeitos na produção. Assim, para uma elasticidade oferta menor para as mulheres, o empregador, ao maximizar os lucros, igualando o custo de contratação dos homens com o das mulheres oferecendo um salário menor para elas, estaria gerando uma discriminação.

A segunda categoria é a do preconceito estatístico, no qual os empregadores projetam nos indivíduos algumas características observadas no grupo. Esse comportamento se justifica por causa das dificuldades para o empregador em obter informações necessárias sobre a produtividade dos potenciais candidatos. As principais referências para esse tipo de discriminação são os estudos de Phelps (1972), Spence (1973) e Aigner e Cain (1977). O exemplo mais comum é a situação em que duas pessoas, um homem e uma mulher, estão concorrendo a uma vaga numa determinada empresa e, na seleção, o curriculum vitae e a entrevista não permitiram prever perfeitamente a verdadeira produtividade dos candidatos. A incerteza leva o empregador a utilizar as estatísticas sobre a performance média do grupo ao qual pertence cada candidato para prever a sua produtividade. Dessa forma, o candidato que pertence ao grupo com maior produtividade beneficia-se gerando uma discriminação estatística.

Por fim, a terceira categoria está relacionada aos gostos e preferências, ou seja, o preconceito é pessoal. Os empregadores, empregados ou consu- 
midores não aceitam o convívio no ambiente de trabalho com funcionário ou mesmo vendedor pertencente a um grupo de características específicas, como, por exemplo, de sexo ou de raça. Merece destaque o estudo The economics of discrimination de 1957, escrito por Gary Becker e que se tornou referência sobre o tema. Em sua abordagem, a preferência por discriminar é incorporada dentro da função utilidade dos agentes, podendo ter origem nos empregadores, nos empregados e nos consumidores.

Para uma melhor compreensão, vejamos o caso particular do empregador. Para esse, o comportamento está inversamente relacionado aos lucros das firmas uma vez que ao agir dessa maneira, demonstra que ele está disposto a pagar, seja direta ou indiretamente, na forma de uma redução nos lucros, por estar associado a algum grupo ao invés de outro. Num mercado competitivo, essa situação não deveria persistir porque as firmas que não discriminam teriam vantagens ao contratar os trabalhadores discriminados, oferecendo-lhes menores salários se comparados aos pagos pelas firmas discriminatórias. Assim, trata-se de uma situação que não deveria perdurar por muito tempo.

É importante observar que nessa categoria se encontra a maior parte dos estudos empíricos sobre discriminação. Nela se integra o presente trabalho, em cujo modelo utilizado para mensurar os diferenciais de salários por gênero na indústria avícola da Região Sul do Brasil está implícita a hipótese da discriminação de um grupo de indivíduos que possuem produtividades iguais, mas recebem salários diferentes ou tratamento diferenciado, seja por causa de sua raça, sexo ou origem, sem que tais características tenham efeito sobre sua produtividade. Assim, após fazer um breve resumo sobre como a discriminação vem sendo tratada pelos economistas, a seguir é descrito o procedimento empregado na sua mensuração.

\section{Mensurando discriminação}

Os primeiros trabalhos sobre discriminação, seja de caráter racial, seja mesmo de gênero, partiram de uma equação de rendimentos em que se considerava o salário como uma função linear de $\boldsymbol{k}$ variáveis associadas à produtividade da mão-de-obra e de uma variável relacionada à produtividade para a raça ou sexo. No caso específico de identificar os diferenciais de salários por gênero, essa relação era expressa pela equação: 


$$
\ln w_{j}=\alpha+\gamma Z_{j}+\sum_{l=1}^{k} \beta_{l} X_{l j}+u_{j}(j=1, \ldots ., N)
$$

onde: $\boldsymbol{l n} w_{j}=$ logaritmo neperiano do salários; $\boldsymbol{X}_{\boldsymbol{l}}$, = variáveis explicativas associadas à produtividade da mão-de-obra $\operatorname{com} l=1, \ldots . ., k$; $\boldsymbol{Z}=$ variável dummy em que $\boldsymbol{Z}=\mathbf{1}$ para homens e $\boldsymbol{Z}=\boldsymbol{0}$ para as mulheres; $\boldsymbol{u}$ são os erros aleatórios que obedecem às hipóteses estatísticas usuais e $\alpha, \gamma$, e $\beta$ são parâmetros. Uma vez estimada a equação (1), as mulheres são discriminadas se o coeficiente $\gamma$ for positivo.

Embora amplamente utilizada na literatura, com o passar do tempo essa maneira de mensurar discriminação deu lugar à outra forma. Seguindo a formalização de Borjas (1996), suponham-se dois grupos de trabalhadores que possam ser definidos como de homens e de mulheres. Considere-se que o salário médio dos homens é dado por $\bar{w}_{h}$, ao passo que o salário médio das mulheres é dado por $\bar{w}_{m}$. Assim, a definição de discriminação pode ser dada pela diferença entre os salários médios.

$$
\Delta \bar{w}=\ln \bar{w}_{h}-\ln \bar{w}_{m}
$$

Apesar de a equação (2) se mostrar intuitiva, ela apresenta alguns problemas, pois existem outros fatores que geram diferenças salariais entre os homens e mulheres. Por exemplo, os homens podem possuir um maior nível de escolaridade. Segundo a teoria do capital humano, quanto maior o investimento em educação e qualificação, maior deverá ser o retorno obtido; neste caso, maiores deverão ser os salários. Portanto, "this definition is unappealing because it is comparing apples and oranges” (Borjas, 1996, p. 336).

Assim, uma definição apropriada de discriminação no mercado de trabalho deveria comparar o salário de trabalhadores que têm o mesmo nível de qualificação. Esse ajuste poderia ser obtido por meio das estimações de regressões que relacionam os rendimentos dos homens ou mulheres a características socioeconômicas e habilidades. Dessa forma, as equações de salários estimadas separadamente para mulheres e homens avaliadas na média poderiam ser expressas por: 
- função de rendimentos dos homens: $\ln \bar{w}_{h}=\hat{\beta}_{h 0}+\sum_{j=1}^{N} \bar{X}_{h}^{j} \hat{\beta}_{h}^{j}$

- função de rendimentos das mulheres: $\ln \bar{w}_{m}=\hat{\beta}_{m 0}+\sum_{j=1}^{N} \bar{X}_{m}^{j} \hat{\beta}_{m}^{j}$

Onde $\boldsymbol{l n} \bar{w}_{i}$ é o logaritmo do salário médio e $\boldsymbol{i}=\boldsymbol{h}, \boldsymbol{m}, \hat{\beta}_{i 0}$ é o coeficiente estimado do intercepto; $\hat{\beta}_{i}^{j}$ é um vetor coluna dos coeficientes de inclinação estimados para j-ésimo regressores e $\bar{X}_{i}^{j}$ é um vetor linha da média dos j-ésimo regressores. Além disso, existem $\mathrm{N}$ variáveis definidas para $\mathrm{N}$ conjunto de regressores.

A interpretação dos coeficientes apresentados nas equações (4) e (5) é direta. O coeficiente $\hat{\beta}_{h}^{j}$ nos mostra o quanto um homem recebe a mais, caso ele obtenha um ano a mais de escolaridade, ao passo que o coeficiente $\hat{\beta}_{m}^{j}$ dá a mesma estatística para uma mulher. Se, para os empregadores, a educação adquirida pela mulher vale tanto quanto a educação adquirida por homens, esses dois coeficientes seriam iguais (então $\hat{\beta}_{h}^{\text {esc }}=\hat{\beta}_{m}^{\text {esc }}$ ). Similarmente, os interceptos $\hat{\beta_{h 0}}$ e $\hat{\beta_{m 0}}$ dão o nível do perfil de rendimentos para cada um dos dois grupos. Se, para os empregadores, a utilidade da habilidade de homens e mulheres que têm zero de escolaridade é igual, os interceptos seriam os mesmos (ou $\hat{\beta}_{h 0}=\hat{\beta}_{m 0}$ ). Portanto, o modelo de regressão implica que o diferencial de salário bruto pode ser descrito, dadas as propriedades dos mínimos quadrados ordinários, por:

$$
\ln \bar{w}_{h}-\ln \bar{w}_{m}=\hat{\beta}_{h 0}+\sum_{j=1}^{N} \bar{X}_{h}^{j} \hat{\beta}_{h}^{j}-\hat{\beta}_{m 0}-\sum_{j=1}^{N} \bar{X}_{m}^{j} \hat{\beta}_{m}^{j} .
$$

onde $\bar{X}_{h}$ dá a escolaridade média do homem e $\bar{X}_{m}$, a escolaridade média da mulher. Ao somar e subtrair o lado direito da equação (6) o termo $\sum_{j=1}^{N} \bar{X}_{m}^{(j)^{\prime}} \hat{\beta}_{h}^{(j)}$, pode-se decompor o diferencial de salário bruto entre homens e mulheres segundo a seguinte equação:

$$
\ln \bar{w}_{h}-\ln \bar{w}_{m}=\underbrace{\left(\hat{\beta}_{h 0}-\hat{\beta}_{m 0}\right)+\sum_{j=1}^{N} \bar{X}_{m}^{(j)^{\prime}} \Delta \hat{\beta}^{(j)}}_{\text {discrim min } \text { ac } \tilde{a} o^{\circ}}+\underbrace{\sum_{j=1}^{N} \Delta \bar{X}^{(j)^{\prime}} \hat{\beta}_{h}^{(j)}}_{\text {habilidades }}
$$


onde $\Delta \hat{\beta}^{(j)}=\hat{\beta}_{h}^{j}-\hat{\beta}_{m}^{j}$ e $\Delta \bar{X}^{(j)}=\bar{X}_{h}^{(j)}-\bar{X}_{m}^{(j)}$.

A equação (7) é conhecida como a decomposição de Oaxaca e é muito utilizada por mostrar que o diferencial de salário bruto consiste em duas partes: uma parcela que surge porque homens e as mulheres, em média, possuem diferentes habilidades, ao passo que a outra é atribuída à discriminação do mercado de trabalho. No caso em que os homens e mulheres possuem a mesma escolaridade média (ou $\Delta \bar{X}^{(j)}=\bar{X}_{h}^{(j)}-\bar{X}_{m}^{(j)}=0$ ), o segundo termo da equação (7) será zero e o resultado será um diferencial bruto devido apenas à discriminação. Dessa forma, uma parte do diferencial de salários brutos surgirá quando os dois grupos possuírem diferentes habilidades.

É importante ressaltar que, apesar de ter sido empregada apenas a escolaridade para apresentar a decomposição de Oaxaca, o modelo pode ser estendido para incluir as variáveis como idade, experiência, duração do emprego, entre outras, visando controlar, dessa forma, todas as dimensões nas quais as habilidades dos grupos diferem. Caso exista alguma característica que porventura possa afetar os rendimentos e que não se encontre no modelo, a mensuração da discriminação não estará correta.

No presente trabalho, além da escolaridade, consideraram-se como variáveis explicativas a idade, o duração do emprego e a ocupação dentro da empresa. Em virtude de as informações sobre escolaridade e duração do emprego serem discretas, algumas alterações devem ser feitas para adequar a equação de salários e a decomposição de Oaxaca ao uso de variáveis binárias (dummies).

Assim, considere-se o caso em que a variável $\boldsymbol{V}$ é definida como um conjunto de variável dummy inserida na equação de salários. O conjunto de valor médio da variável dummy é denotado por $\left\{V_{i k} \mid k=1, \ldots \ldots \ldots . . K_{1}\right\}$ em que $\sum_{k=1}^{K_{1}} \bar{V}_{\boldsymbol{k}}=1$ e $\boldsymbol{i}=\boldsymbol{h}, \boldsymbol{m}$. Sem perda de generalidade, a primeira categoria da variável dummy $\left(\bar{V}_{i 1}\right)$ excluída servirá como o grupo de referência. As equações de salários estimadas para os homens e para as mulheres na média podem ser expressas como:

$$
\begin{aligned}
\ln \bar{w}_{h} & =\hat{\beta}_{h 0}+\sum_{j=1}^{N} \bar{V}_{h k} \hat{\delta}_{h k}+\sum_{j=1}^{N} \bar{X}_{h}^{(j)^{\prime}} \hat{\beta}_{h}^{(j)} \\
& =\sum_{j=1}^{N} \bar{V}_{h k} \hat{\theta}_{h k}+\sum_{j=1}^{N} \bar{X}_{h}^{(j)^{\prime}} \hat{\beta}_{h}^{(j)}
\end{aligned}
$$




$$
\begin{aligned}
\ln \bar{w}_{m} & =\hat{\beta}_{m 0}+\sum_{j=1}^{N} \bar{V}_{m k} \hat{\delta}_{m k}+\sum_{j=1}^{N} \bar{X}_{m}^{(j)^{\prime}} \hat{\beta}_{m}^{(j)} \\
& =\sum_{j=1}^{N} \bar{V}_{m k} \hat{\theta}_{m k}+\sum_{j=1}^{N} \bar{X}_{m}^{(j)^{\prime}} \hat{\beta}_{m}^{(j)}
\end{aligned}
$$

onde $\hat{\delta_{i k}}=\hat{\theta_{i k}}-\hat{\theta_{i 1}}$. Normalizando o grupo de referência excluído como $\hat{\beta}_{i 0}=\hat{\theta}_{i 1}$ a decomposição de Oaxaca para salário passa a ser dada por:

$\ln \bar{w}_{h}-\ln \bar{w}_{m}=\underbrace{\sum_{k=2}^{K_{1}} \bar{V}_{m k}\left(\hat{\theta}_{h k}-\hat{\theta}_{m k}\right)+\sum_{j=1}^{N} \Delta \bar{X}_{m}^{(j)} \Delta \hat{\beta}^{(j)}}_{\text {driscri min } a \text { aca }}+\underbrace{\sum_{k=1}^{K_{1}}\left(\bar{V}_{h k}-\bar{V}_{m k}\right) \hat{\theta}_{h k}+\sum_{j=1}^{N} \Delta \bar{X}^{(j)^{\prime}} \hat{\beta}_{h}^{(j)}}_{\text {habilitades }}$

Após descrever como pode ser mensurada a discriminação com base na decomposição de Oaxaca, na seqüência será apresentada a análise dos dados e os resultados das equações de salários da indústria de aves da região Sul do Brasil.

\section{Resultados e discussão}

Nesta seção apresentam-se a descrição dos dados utilizados para mensurar os diferenciais de salários por gênero, os resultados obtidos na estimação das equações de determinação dos salários e a decomposição de Oaxaca (1973).

\subsection{Descrição dos dados}

Para analisar os diferenciais de salários por gênero na indústria avícola da região Sul do Brasil são utilizados os dados da Relação Anual de Informação Social (RAIS) para o ano de 1998, disponibilizado pela base de dados do Ministério de Emprego e Trabalho. O número total de trabalhadores dessa indústria corresponde a 31.586 indivíduos com mais de 13 anos de idade, que, separados por gênero, perfazem um total de 21.236 homens e 10.350 mulheres. Restringindo esse número para uma amostra que incorporasse apenas a ocupação magarefe obtém-se um total de 12.343 indivíduos, correspondendo a 39,07\% do total de trabalhadores, dos quais 7.248 são homens e 5.095, mulheres. As variáveis utilizadas 
na análise compreendem os salários (média anual do número de salários mínimos), gênero, idade, ocupação, escolaridade e duração do emprego, que indica quanto tempo o empregado está trabalhando na firma.

Com relação a essas duas últimas variáveis, é importante mencionar que quando do preenchimento da RAIS, a informação correspondente ao nível de educação do trabalhador é dividida em intervalos que permitem classificá-lo como sem instrução, com primeiro grau incompleto, com primeiro grau completo, com segundo grau incompleto, com segundo grau completo, com superior incompleto e, por fim, com superior completo. Para duração do emprego ou tempo de emprego $(t)$, os intervalos correspondem até 2,9 meses, de 3 a 5,9 meses, de 6 a 11,9 meses, de 12 a 23,9 meses, de 24 a 35,9 meses, de 36 a 59,9 meses, de 60 a 119,9 meses e de 120 ou mais meses. Como essas informações são discretas, utilizou-se variáveis binárias para identificar os intervalos.

A Tabela 1 apresenta as médias obtidas para os salários, para a idade, para cada nível de escolaridade e duração do emprego para a indústria e para amostra de magarefes separados por gênero. De imediato, pela inspeção das médias dos trabalhadores da indústria avícola, é possível verificar que os homens possuem uma média mais elevada para a variável salários, sugerindo que recebem, em média, um salário mais elevado em relação ao das mulheres. Outra variável que apresenta uma média maior para eles é a escolaridade a partir do $1^{\circ}$. grau completo. Conjuntamente, esses dois resultados evidenciam um aspecto relevante para a presente análise, já que a teoria do capital humano postula que, quanto maior o nível de investimento em educação e qualificação, maior deverá ser o retorno.

Com respeito à variável idade, observa-se que a média apresentada é maior para as mulheres. Para a duração do emprego, em que as médias ora são maiores para os homens, ora para as mulheres, não é possível identificar nenhum padrão. Apenas chama atenção o fato de que, para a duração do emprego de até 2,9 meses, de 3 a 5,9 meses, de 6 a 11,9 meses e de 12 a 23,9 meses, ou seja, intervalos de menor duração, os homens têm as maiores médias. Esse resultado, associado à evidência de que os homens possuem maior média de escolaridade, sugere que os trabalhadores do sexo masculino apresentam uma maior rotatividade, uma vez que, quanto maior a qualificação, maiores serão as oportunidades de emprego. No entanto, para se comprovar tal hipótese seria necessá- 
rio estender a análise para mais anos e fazer um acompanhamento dos trabalhadores nessa indústria.

Tabela 1 - Descrição da média dos rendimentos, idade, escolaridade e duração do emprego

\begin{tabular}{l|ccc|ccc}
\hline \multirow{2}{*}{\multicolumn{1}{c|}{ Variável }} & \multicolumn{3}{c|}{ Indústria } & \multicolumn{3}{c}{ Magarefe } \\
\cline { 2 - 7 } & Homem & Mulher & Total & Homem & Mulher & Total \\
\hline Salários & 2,8913 & 2,5626 & 2,7836 & 2,7766 & 2,6406 & 2,7205 \\
Idade & 28,2457 & 29,5071 & 28,6590 & 27,8972 & 29,4066 & 28,5202 \\
Sem instrução & 0,0065 & 0,0087 & 0,0072 & 0,0104 & 0,0131 & 0,0115 \\
$1^{\circ}$. grau incompleto & 0,5540 & 0,6399 & 0,5821 & 0,7428 & 0,7173 & 0,7323 \\
$1^{\circ}$. grau completo & 0,1718 & 0,1471 & 0,1637 & 0,1258 & 0,1244 & 0,1252 \\
$2^{\circ}$. grau incompleto & 0,1387 & 0,0952 & 0,1245 & 0,0579 & 0,0631 & 0,0601 \\
$2^{\circ}$. grau completo & 0,1197 & 0,1035 & 0,1144 & 0,0605 & 0,0787 & 0,0680 \\
Superior incompleto & 0,0048 & 0,0035 & 0,0044 & 0,0015 & 0,0023 & 0,0018 \\
Superior completo & 0,0042 & 0,0017 & 0,0034 & 0,0008 & 0,0007 & 0,0008 \\
Até 2,9 meses & 0,1001 & 0,0743 & 0,0916 & 0,1008 & 0,0708 & 0,0884 \\
3 a 5,9 meses & 0,0852 & 0,0643 & 0,0784 & 0,0684 & 0,0370 & 0,0554 \\
6 a 11,9 meses & 0,1406 & 0,1191 & 0,1336 & 0,1376 & 0,1224 & 0,1314 \\
12 a 23,9 meses & 0,1322 & 0,1076 & 0,1242 & 0,1499 & 0,1105 & 0,1336 \\
24 a 35,9 meses & 0,0940 & 0,1074 & 0,0984 & 0,1055 & 0,1063 & 0,1058 \\
36 a 59,9 meses & 0,1743 & 0,2294 & 0,1924 & 0,1937 & 0,2376 & 0,2118 \\
60 a 119,6 meses & 0,1796 & 0,2151 & 0,1913 & 0,1767 & 0,2282 & 0,1980 \\
120 ou mais meses & 0,0935 & 0,0824 & 0,0898 & 0,0670 & 0,0867 & 0,0751 \\
N & 21.236 & 10.350 & 31.586 & 7.248 & 5.095 & 12.343 \\
Participação (\%) & 67.23 & 32.77 & 100.00 & 58.72 & 41.28 & 100.00 \\
\hline
\end{tabular}

Fonte: Cálculos do autor.

As médias para a amostra dos trabalhadores que desempenham a função de magarefe mostram que o salário médio percebido pelos homens é maior do que os recebidos pelas mulheres. Estas, possuem idade média superior à apresentada pelos homens e mostram-se mais qualificadas, quando se observam os níveis mais elevados de educação, ou seja, a partir do $2^{\circ}$ grau incompleto. Além disso, possuem médias mais elevadas de duração do emprego a partir do intervalo que corresponde de 24 a 35,9 meses.

Esses resultados, se comparados àqueles apresentados para a indústria como um todo (ver coluna "total" na Tabela 1) mostram significativas 
diferenças, como, por exemplo, os homens possuem maior escolaridade média e salário médio para o total de trabalhadores. Por outro lado, as mulheres que exercem a ocupação de magarefe apresentam um salário médio maior àquele observado para a indústria como um todo. Em termos percentuais, 41,28\% dos magarefes são trabalhadores do sexo feminino, ao passo que, para o total de trabalhadores da indústria, esse percentual se reduz para $34,77 \%$. Dessa forma, faz sentido admitir uma maior preferência por mão-de-obra feminina quando se trata dessa ocupação.

Antes de passar para os resultados das estimações da equação de determinação dos salários, uma análise dos ganhos relativos dos trabalhadores mais educados para os magarefes, tanto em termos de massa salarial quanto em termos de salários relativos, com a finalidade de analisar a parcela do emprego que é absorvida por esses trabalhadores, pode contribuir para uma melhor descrição dos dados.

Para tanto, define-se $\boldsymbol{w} \mathbf{1}$ como o logaritmo do salário médio das pessoas que possuem o $1^{\circ}$. grau completo (não qualificados), o que perfaz oito anos de estudos. Definindo $w 2$ como sendo o salário médio das pessoas que possuem o $2^{\circ}$. grau completo (qualificados), o que representa um total de 11 anos de estudo e considerando $\boldsymbol{n} \mathbf{1}$ e $\boldsymbol{n} \mathbf{2}$ como o número das pessoas que têm essas duas características, podem-se obter os ganhos relativos dos trabalhadores mais educados em termos de massa salarial (PED) e em termos da parcela do emprego que é absorvida por esses trabalhadores (NED):

$$
\begin{aligned}
& \text { PED }=\frac{(w 2 * n 2)}{(w 1 * n 1)+(w 2 * n 2)} \\
& \mathrm{e} \\
& N E D=\frac{n 2}{(n 1+n 2)}
\end{aligned}
$$

Considerando essas variáveis e a relação entre salários relativos dos qualificados (w2/w1) e de sua freqüência relativa $(n 2 / n 1)$, a seguir apresentam-se os ganhos por gênero. 
Tabela 2 - Ganhos e freqüências relativas por gênero para os magarefes

\begin{tabular}{lccc}
\hline \multicolumn{1}{c}{ Variáveis } & Homens & Mulheres & Total da amostra \\
\hline PED & 0,3482 & 0,3895 & 0,3656 \\
NED & 0,3249 & 0,3874 & 0,3521 \\
PED/NED & 1,0717 & 1,0054 & 1,0383 \\
w2/w1 & 1,1098 & 1,0088 & 1,0609 \\
n2/n1 & 0,4814 & 0,6325 & 0,5433 \\
\hline
\end{tabular}

Fonte: Cálculos do autor.

A Tabela 2 apresenta os ganhos e freqüências relativas por gênero para os magarefes. Chama a atenção que as mulheres possuem, em termos relativos, a maior parcela de pessoas qualificadas (NED) e que a parcela de salários para elas (PED) é 11,86\% maior que a verificada nos homens. Esse resultado mostra-se interessante porque, no grupo dos homens, menos pessoas parecem ganhar mais relativamente ao observado no grupo das mulheres. Se comparada essa informação com a obtida para o total da amostra, verifica-se que é ainda menor.

Esse indício pode ser mais bem observado pela razão entre PED e NED, que mostra a proporção entre a parcela do salário destinada aos trabalhadores qualificados e sua parcela no emprego. Para os homens, essa relação é de 1,0717, superando os 1,0054 obtidos para as mulheres. Essa relação supera, ainda, o resultado encontrado para o total da amostra, permitindo afirmar que a parcela da massa salarial que vai para os qualificados é maior para os homens do que no grupo das mulheres.

Outra evidência que vem reforçar esse argumento pode ser obtida quando se observa a relação dos salários entre os qualificados e não qualificados. Para os homens essa relação é de 1,1098 vezes, ao passo que, para as mulheres, chega a 1,0088 vezes e, novamente, o resultado obtido dessa relação para os homens supera aquele encontrado para o total da amostra. De qualquer forma, essas estimativas sugerem que a possibilidade de diferenciais de salários favoráveis para as mulheres, como era imaginado, não ocorre, mesmo se sabendo que as empresas que compõem a indústria avícola ofertam vagas para essa ocupação tendo em vista a contratação de mulheres.

Assim, após fazer uma análise descritiva dos dados e de realizar uma análise dos ganhos por um nível educacional escolhido, a seguir 
são apresentados os resultados obtidos na estimação da equação de determinação de salários e a decomposição de Oaxaca.

\subsection{Equações de salários e a decomposição de Oaxaca}

Para a determinação de salários utilizou-se um total de 12.343 informações individuais e estimou-se a equação (1), a qual incorpora uma variável binária para sexo que assume valores $\mathrm{Z}=1$ para homens e $\mathrm{Z}$ $=0$ para mulheres e têm a finalidade de verificar se as mulheres são discriminadas. Em virtude do uso de variáveis binárias para descrever melhor os intervalos do nível de escolaridade e duração do emprego, optou-se pela retirada dos intervalos que correspondem aos trabalhadores sem instrução e aos trabalhadores com duração do emprego até 2,9 meses, os quais servirão como grupo de referência. Os coeficientes estimados são interpretados como a diferença percentual entre o salário esperado da categoria para a qual a variável binária assume valor 1 vis a vis o salário da categoria tomada como base, grupo de referência. Além dessas variáveis, utilizaram-se a idade e idade ao quadrado como uma proxy para captar a contribuição do treinamento e experiência dos trabalhadores.

A Tabela 3 apresenta os resultados para MQO e MQO robustos à heterocedasticidade e, como pode ser observado, os coeficientes obtidos para a equação (1) possuem os sinais esperados. O coeficiente de idade2 é menor do que zero e evidencia uma relação côncava entre o salário e a idade, ou seja, são indícios de retornos decrescentes para idade. Os coeficientes das variáveis binárias responsáveis em captar os níveis de instrução e duração do emprego mostraram-se positivos e crescentes. Além disso, esses resultados mostram que o retorno da educação e da experiência não é linear, uma vez que, quanto maior a educação e a experiência, maiores são os retornos. Os coeficientes estimados são estatisticamente significativos e o teste F para essa estimação permite inferir a rejeição da hipótese nula em que todos os coeficientes são iguais a zero. 
Tabela 3 - Resultados da equação salários - ocupação de magarefe

\begin{tabular}{|c|c|c|c|c|}
\hline \multirow{2}{*}{ Variáveis } & \multicolumn{2}{|c|}{ MQO } & \multicolumn{2}{|c|}{ MQO robusto à heterocedasticidade } \\
\hline & Coeficiente & Desvio padrão & Coeficiente & Desvio padrão \\
\hline Constante & 0,2653 & $(0,0280)^{\mathrm{C}}$ & 0,2653 & $(0,0303)^{\mathrm{C}}$ \\
\hline Idade & 0,0120 & $(0,0014)^{\mathrm{C}}$ & 0,0120 & $(0,0016)^{\mathrm{C}}$ \\
\hline Idade $^{2}$ & $-0,0172$ & $(0,0020)^{\mathrm{C}}$ & $-0,0172$ & $(0,0025)^{\mathrm{C}}$ \\
\hline $1^{\circ}$. grau incompleto & 0,1222 & $(0,0172)^{\mathrm{C}}$ & 0,1222 & $(0,0186)^{\mathrm{C}}$ \\
\hline $1^{\circ}$. grau completo & 0,1315 & $(0,0179)^{\mathrm{C}}$ & 0,1315 & $(0,0195)^{\mathrm{C}}$ \\
\hline $2^{\circ}$. grau incompleto & 0,1296 & $(0,0188)^{\mathrm{C}}$ & 0,1296 & $(0,0200)^{\mathrm{C}}$ \\
\hline $2^{\circ}$. grau completo & 0,2118 & $(0,0186)^{\mathrm{C}}$ & 0,2118 & $(0,0208)^{\mathrm{C}}$ \\
\hline Superior incompleto & 0,4986 & $(0,0457)^{\mathrm{C}}$ & 0,4986 & $(0,1077)^{\mathrm{C}}$ \\
\hline Superior completo & 0,5139 & $(0,0665)^{\mathrm{C}}$ & 0,5139 & $(0,2208)^{\mathrm{C}}$ \\
\hline 3 a 5,9 meses & 0,1479 & $(0,0099)^{\mathrm{C}}$ & 0,1479 & $(0,0086)^{\mathrm{C}}$ \\
\hline 6 a 11,9 meses & 0,1861 & $(0,0079)^{\mathrm{c}}$ & 0,1861 & $(0,0076)^{\mathrm{C}}$ \\
\hline 12 a 23,9 meses & 0,2637 & $(0,0079)^{\mathrm{C}}$ & 0,2637 & $(0,0078)^{\mathrm{C}}$ \\
\hline 24 a 35,9 meses & 0,3089 & $(0,0084)^{\mathrm{c}}$ & 0,3089 & $(0,0087)^{\mathrm{C}}$ \\
\hline 36 a 59,9 meses & 0,3733 & $(0,0075)^{\mathrm{C}}$ & 0,3733 & $(0,0076)^{\mathrm{C}}$ \\
\hline 60 a 119,6 meses & 0,5117 & $(0,0077)^{\mathrm{C}}$ & 0,5117 & $(0,0080)^{\mathrm{C}}$ \\
\hline 120 ou mais meses & 0,6735 & $(0,0096)^{\mathrm{C}}$ & 0,6735 & $(0,0117)^{\mathrm{C}}$ \\
\hline $\mathrm{Z}$ & 0,0759 & $(0,0037)^{\mathrm{C}}$ & 0,0759 & $(0,0038)^{\mathrm{C}}$ \\
\hline $\mathrm{R}^{2}$ ajustado & 0,43 & & 0,44 & \\
\hline $\mathrm{F}(16,12326)$ & 605,88 & & 507,43 & \\
\hline $\mathrm{N}$ & 12.343 & & 12.343 & \\
\hline SQR & 509,31 & & 509,31 & \\
\hline Teste Cook-Weisberg 1 & 514,61 & & & \\
\hline
\end{tabular}

Fonte: Cálculos do autor

Obs.: (a) significante a $10 \%$, (b) significante a $5 \%$ e (c) significante a $1 \%$.

(1) O Teste Cook-Weisberg possui Ho: variância constante.

A variável dummy Z incluída para verificar a existência de diferenças entre homens e mulheres mostra-se estatisticamente significativa e com sinal esperado. Embora esse resultado permita detectar as diferenças entre homens e mulheres, outros coeficientes além do intercepto deveriam captar informações sobre a discriminação. Esse problema pode ser resolvido a partir de um ajuste das equações por sexo. Para tanto, a amostra total de magarefes foi dividida em dois grupos, sendo o primeiro composto apenas por mulheres e o segundo, por homens. Tal procedimento permite estimar as equações (8) e (9) para determinar salários 
em que as variáveis explicativas são as mesmas utilizadas na equação (1) exceto a variável binária para sexo.

A Tabela 4 apresenta os resultados obtidos para a equação (8) e (9) estimadas. Como pode ser observado, os coeficientes estimados das variáveis que compõem a amostra de mulheres apresentaram sinal esperado e são estatisticamente significativos ao nível de 1 e $5 \%$ de significância, exceto para variáveis idade e idade2. Para a amostra composta apenas por homens, os resultados não foram muito diferentes. Os coeficientes estimados possuem o sinal esperado e foram todos significativos, com um $\mathrm{R}^{2}$ igual a 0,47 e o teste $\mathrm{F}$ igual a 365,55 .

Em suma, os resultados obtidos pelas equações (1), (8) e (9) estão de acordo com aquele esperado pela teoria. A inclusão da variável duração do emprego mostrou-se um importante determinante no salário percebido pelos magarefes já que, quanto mais tempo os trabalhadores permanecem no emprego, maior é o salário recebido da empresa. Com respeito à escolaridade, cuja variável, segundo a teoria do capital humano, é responsável por explicar os diferenciais de salários existentes entre os trabalhadores, os resultados encontrados mostram que aqueles que possuem maior nível de escolaridade dentro de cada amostra (homem e mulher) percebem um maior retorno da educação.

Comparativamente, os retornos da educação para as mulheres são maiores em relação aos apresentados para os trabalhadores do sexo masculino, exceto para aqueles que têm o nível superior incompleto e completo. O oposto pode ser observado para os retornos da variável tempo de emprego, em que os homens apresentam maiores retornos a partir do intervalo de 6 a 11,9 meses de duração no emprego.

Antes de apresentar os resultados para a decomposição de Oaxaca, uma análise gráfica da relação entre salário médio e escolaridade e salário médio e duração do emprego permite obter informações acerca da existência de diferenciais de salários por gênero. O Gráfico 1, elaborado com base em dados brutos e que apresenta a relação entre salário médio e escolaridade mostra evidências de diferenças salariais entre homens e mulheres. Observa-se que as diferenças são favoráveis aos homens, mas se reduzem à medida que aumenta o nível de escolaridade. Para os trabalhadores que possuem o segundo grau incompleto, não há diferenças salariais por gênero. Contudo, a partir desse nível de escolaridade as diferenças tendem a ser maiores e favoráveis aos homens. 
Tabela 4 - Resultados da equação de salários por gênero ocupação de magarefe

\begin{tabular}{|c|c|c|c|c|}
\hline \multirow{2}{*}{ Variável } & \multicolumn{2}{|c|}{ Homens } & \multicolumn{2}{|c|}{ Mulheres } \\
\hline & Coeficientes & Desvio padrão & Coeficientes & Desvio padrão \\
\hline Constante & 0,2723 & $(0,0430)^{\mathrm{C}}$ & 0,4309 & $(0,0403)^{\mathrm{C}}$ \\
\hline Idade & 0,0178 & $(0,0023)^{\mathrm{C}}$ & 0,0008 & $(0,0021)$ \\
\hline Idade2 & $-0,0249$ & $(0,0037)^{\mathrm{C}}$ & $-0,0022$ & $(0,0032)$ \\
\hline $1^{\circ}$. grau incompleto & 0,0839 & $(0,0272)^{\mathrm{C}}$ & 0,1630 & $(0,0235)^{\mathrm{C}}$ \\
\hline $1^{\circ}$. grau completo & 0,0939 & $(0,0283)^{\mathrm{c}}$ & 0,1689 & $(0,0249)^{\mathrm{C}}$ \\
\hline $2^{\circ}$. grau incompleto & 0,0965 & $(0,0292)^{\mathrm{C}}$ & 0,1602 & $(0,0255)^{\mathrm{c}}$ \\
\hline $2^{\circ}$. grau completo & 0,1952 & $(0,0309)^{\mathrm{c}}$ & 0,2158 & $(0,0251)^{\mathrm{C}}$ \\
\hline Superior incompleto & 0,8011 & $(0,1594)^{\mathrm{c}}$ & 0,1845 & $(0,0490)^{\mathrm{c}}$ \\
\hline Superior completo & 0,6955 & $(0,3423)^{\mathrm{C}}$ & 0,2259 & $(0,1001)^{\mathrm{C}}$ \\
\hline 3 a 5,9 meses & 0,1455 & $(0,0104)^{\mathrm{C}}$ & 0,1646 & $(0,0151)^{\mathrm{C}}$ \\
\hline 6 a 11,9 meses & 0,1819 & $(0,0094)^{\mathrm{C}}$ & 0,1955 & $(0,0126)^{\mathrm{C}}$ \\
\hline 12 a 23,9 meses & 0,2697 & $(0,0094)^{\mathrm{C}}$ & 0,2558 & $(0,0135)^{\mathrm{C}}$ \\
\hline 24 a 35,9 meses & 0,3106 & $(0,0110)^{\mathrm{C}}$ & 0,3010 & $(0,0141)^{\mathrm{C}}$ \\
\hline 36 a 59,9 meses & 0,3742 & $(0,0097)^{\mathrm{C}}$ & 0,3655 & $(0,0123)^{\mathrm{C}}$ \\
\hline 60 a 119,6 meses & 0,5238 & $(0,0103)^{\mathrm{C}}$ & 0,4929 & $(0,0129)^{\mathrm{C}}$ \\
\hline 120 ou mais meses & 0,7174 & $(0,0167)^{\mathrm{C}}$ & 0,6164 & $(0,0158)^{\mathrm{C}}$ \\
\hline $\mathrm{R}^{2}$ ajustado & 0,47 & & 0,39 & \\
\hline Teste F & 365,55 & & 205,63 & \\
\hline $\mathrm{N}$ & 7248 & & 5095 & \\
\hline SQR & 311,53 & & 188,22 & \\
\hline
\end{tabular}

Fonte: Cálculos do autor

Obs.: (a) significante a $10 \%$, (b) significante a $5 \%$ e (c) significante a $1 \%$. Os valores entre parêntesis referem-se aos desvios padrões robustos à heterocedasticidade.

O Gráfico 2 apresenta a relação entre salário médio e a duração do emprego. Como pode ser visto, o comportamento entre essas duas variáveis mostra-se como o esperado, ou seja, à medida que aumenta a duração do emprego, maior é o salário percebido pelo trabalhador, seja homem ou mulher. As linhas nunca se cruzam e, independentemente da duração no emprego, os homens sempre têm salários médios superiores. Embora os salários médios pagos para o intervalo de até 2,9 meses de emprego (t1) pelos empregadores aos trabalhadores do sexo masculino e feminino estejam próximos, quando o tempo de emprego aumenta, a diferença entre os salários médios aumenta; a partir do intervalo de 6 a 
11,9 meses de emprego ( $t 3$ ), essa diferença passa a ser maior, isto é, de uma diferença inicial de 3,88\% , em (t 1 ), se chega a atingir 20,28\% no intervalo que corresponde a 120 ou mais meses (t8).

\section{Figura 1}

Fonte: Elaborado pelo autor.

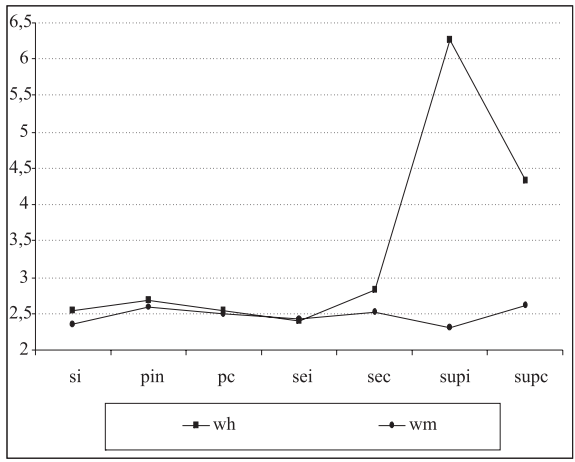

Gráfico 1: Relação entre salário e escolaridade

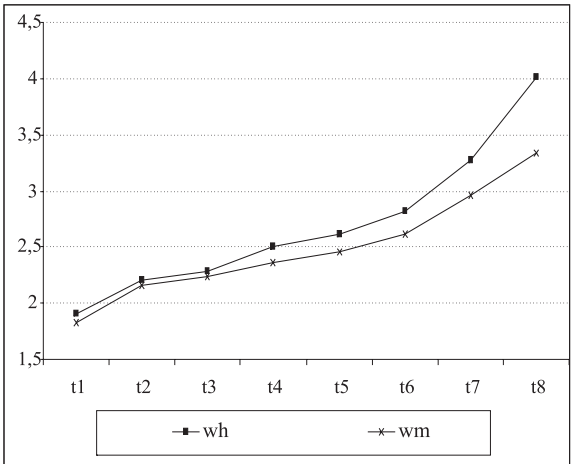

Gráfico 2: Relação entre salário e tempo de emprego

Considerando os resultados das equações de salário para as mulheres e para os homens juntamente com as estatísticas descritivas, pode-se fazer o cálculo da decomposição de Oaxaca. A Tabela 5 apresenta os resultados para a decomposição, observando-se que o diferencial de salário bruto para a ocupação magarefe na indústria avícola é de 0,1660. Como foi mencionado anteriormente, esse diferencial pode ser decomposto em duas partes, sendo que 0,2128 correspondem ao diferencial devido à discriminação e $-0,0468$ correspondem ao diferencial devido à diferença nas características entre homens e mulheres que desempenham a função de magarefe.

Para facilitar a interpretação seria conveniente apresentar os resultados em termos percentuais. Nesse sentido, a discriminação salarial devido ao tratamento é de $128,19 \%$. Desse percentual apenas $28,19 \%$ das diferenças salariais entre homens e mulheres se devem às características da idade, escolaridade e tempo de emprego; o restante deve-se a outros fatores não explicados por características, isto é discriminação no mercado de trabalho. Um leitor não habituado a essa literatura poderia encontrar problemas para compreender as porcentagens negati- 
vas e superiores a $100 \%$. Intuitivamente, o sinal negativo na frente da porcentagem do diferencial devido às características indica (em termos percentuais) que as mulheres deveriam ganhar $28,19 \%$ a mais do que os homens, seja no caso em que a diferença salarial bruta observada fosse zero, seja no caso em que a distribuição de salários entre os dois grupos fosse igual.

Tabela 5 - Decomposição de Oaxaca com controle para ocupação

\begin{tabular}{lccc}
\hline & $\begin{array}{c}\text { Diferença salarial } \\
\text { bruta }\end{array}$ & $\begin{array}{c}\text { Diferencial devido ao } \\
\text { tratamento }\end{array}$ & $\begin{array}{c}\text { Diferencial devido às } \\
\text { características }\end{array}$ \\
\hline Base Mulher & 0,1660 & 0,2128 & $-0,0468$ \\
Total & $(100 \%)$ & $(128,19 \%)$ & $(-28,19 \%)$ \\
\hline
\end{tabular}

Fonte: Cálculos do autor.

A validade desses resultados está condicionada à suposição de que os homens e mulheres diferem quanto as suas características e habilidades. Para verificar se essa suposição é verdadeira emprega-se o Teste de Chow ${ }^{3}$. Trata-se de uma estatística $\hat{F}$ para a hipótese nula de que os coeficientes são iguais entre homens e mulheres. No seu cálculo considera-se a soma dos quadrados dos resíduos para uma regressão restrita $\left(\mathbf{S Q R} \boldsymbol{R}_{R}\right) \mathbf{I}$, que pode ser obtido na Tabela 3, e a soma dos quadrados dos resíduos para uma regressão irrestrita $\left(\boldsymbol{S} \boldsymbol{Q} \boldsymbol{R}_{U N}\right)$, que pode ser obtida pela soma dos resíduos ao quadrado das regressões de determinação de salários para homens e mulheres na Tabela 4.

O teste de Chow calculado para validar a decomposição é $\hat{F}_{(16,12311)}$ $=235,46$ e, considerando a distribuição $\boldsymbol{F}$ para nível de significância de $\alpha=0,01$ e $\alpha=0,05$, verifica-se a rejeição da hipótese nula de igualdade dos coeficientes. A rigor, o resultado obtido pela decomposição de Oaxaca vem ampliar uma série de estudos de natureza empírica que indicaram a existência de diferenciais de salários por gênero.

Os resultados apresentados até aqui mostram que a hipótese inicial de diferenciais de salários favoráveis às mulheres não se verifica, seja por meio das médias dos salários por gênero, seja pelo cálculo da

Para uma exposição didática do teste de Chow, ver Maddala (1992). 
decomposição de Oaxaca. Considerando que a relação entre salários e escolaridade, como mostra a Figura 1, não é tão comportada quando comparada com a relação entre salários e duração do emprego (Figura 1, Gráfico 2) e que a decomposição, segundo Oaxaca e Ranson (1999), é sensível às dummies escolhidas para serem omitidas, optou-se por restringir a amostra de magarefes para uma que contemplasse apenas os trabalhadores com o primeiro grau de escolaridade incompleto.

Tal procedimento se faz necessário por duas razões: a primeira é o fato de que da amostra total de magarefes, $73,23 \%$ encontram-se nessa categoria; segundo, ao admitir que homens e mulheres apresentassem o mesmo nível de escolaridade e ocupassem a mesma função, os diferenciais de salários, caso existissem, não deveriam ser expressivos. As Tabelas 7 e 8 no Apêndice mostram as médias amostrais para o salário, idade e tempo de emprego e o resultado das equações de determinação de salários para os trabalhadores com o primeiro grau de escolaridade incompleto.

Tabela 6 - Decomposição de Oaxaca com controle para ocupação e escolaridade

\begin{tabular}{lccc}
\hline & $\begin{array}{c}\text { Diferença salarial } \\
\text { bruta }\end{array}$ & $\begin{array}{c}\text { Diferencial devido ao } \\
\text { tratamento }\end{array}$ & $\begin{array}{c}\text { Diferencial devido às } \\
\text { características }\end{array}$ \\
\hline Base Mulher & 1,0125 & 1,0472 & $-0,0346$ \\
Total & $(100 \%)$ & $(103,42 \%)$ & $(-3,42 \%)$ \\
\hline
\end{tabular}

Fonte: Cálculos do autor.

A Tabela 6 mostra que o diferencial bruto obtido é de 1,0125. Dessa estimativa 1,0472 correspondem ao diferencial devido à discriminação e -0,0346, às diferenças nas características entre homens e mulheres. Trata-se de um resultado preocupante uma vez que a amostra de trabalhadores é homogênea, isto é, controlada para o nível de escolaridade e ocupação, porém a discriminação apresentou-se maior em termos de diferencial bruto, como pode ser observado ao serem comparados esses resultados com os da Tabelas 5. Em suma, as evidências apresentadas mostram que a discriminação que ocorre no mercado de trabalho como um todo também está presente na indústria avícola. 


\section{5- Conclusão}

O objetivo do presente artigo foi identificar e analisar a extensão dos diferenciais de salários por gênero na indústria avícola da região Sul do Brasil, empregando a decomposição de Oaxaca. A principal motivação para sua realização foi a possibilidade de obter resultados que indicassem a reversão da discriminação salarial do trabalho feminino observada nos estudos realizados para o Brasil. Considerando os trabalhadores no setor de produção que exercem a função de magarefe, os resultados encontrados mostraram que a discriminação existente no mercado de trabalho como um todo também ocorre em relação a esses trabalhadores.

A decomposição de Oaxaca, utilizando os coeficientes estimados pelas equações de rendimentos dos homens e mulheres, demonstra que $28,19 \%$ do diferencial de salários existente devem-se às diferenças nas características entre os homens e mulheres quanto ao nível de escolaridade, idade e tempo de emprego, o que leva a concluir que os primeiros estão em vantagem. Dessa forma, a hipótese inicial de diferenciais de salários a favor das mulheres não é confirmada.

Em termos qualitativos, esse resultado não foi alterado após restringir a amostra total para uma na qual a ocupação de magarefe apenas com primeiro grau incompleto foi considerada. O salário médio entre os dois grupos ainda se manteve favorável aos homens. A duração do emprego mostrou-se importante na determinação dos salários e, certamente, contribuiu para que o grupo de homens mantivesse um salário médio superior ao das mulheres.

A decomposição de Oaxaca calculada com base nos coeficientes das equações de salários estimadas mostrou que o diferencial de salários em razão do tratamento é de 103,42\% e que, dessa estimativa, apenas $3,42 \%$ se devem às características entre os homens e mulheres, sendo o restante apenas devido à discriminação. Ao comparar os resultados encontrados para as duas amostras, verifica-se que o diferencial de salários em virtude das característica é maior para a última. Portanto, mesmo numa situação em que a preferência na contratação de mulheres é levada em conta, os diferenciais de salários continuam favoráveis aos homens. 


\section{6 - Referências bibliográficas}

AIGNER, D. J.; CAIN, G. G. Statistical theories of discrimination in labor market. Industrial in Labor Relations Review, v. 30, p. 175-187, 1977.

ARBACHE, J. S. Determinação e diferencial de salários no Brasil. Desemprego e mercado de trabalho: Ensaios teóricos e empíricos. Viçosa, Editora UFV, p. 125-184, 2000.

AZEVÊDO, J. S. G. de. Relações entre rendimentos e qualificação: explorando os dados da PED/RMS. Revista Econômica do Nordeste, Fortaleza, v. 28, n. especial, p.363-378, julho 1997.

BARROS, R. P.; PONTES, J. P.; VARANDAS, S. Diferenciais de salário: questões metodológicas e ilustrações. Revista de Econometria, Rio de Janeiro, v. VIII, n.2, p.31-53, 1988.

BARROS, R. P.; MENDONÇA, R. S. P. Família e distribuição de renda: o impacto da participação das esposas no mercado de trabalho. Pesquisa e Planejamento Econômico, Rio de Janeiro, v. 19, n. 3, p. 483-504, dezembro 1989.

BECKER, G. The economics of discrimination. Chicago. The University of Chicago Press, 1957.

BERGMAN, B. The effect of white incomes of discrimination in employment. Journal of Political Economy, v. 79, p. 294-313, 1971.

BORJAS, G. J. Labor Economics. New York. Mc Graw-Hill, 1996.

CAIN, G.G. The economic analysis of labor market discrimination: a survey. Handbook of Labor Economics, ed. O. Ashenfelter and R. Layard, v. 1, p. 693-785, 1986.

CAMARGO, J. M.; SERRANO, F. Os dois mercados: homens e mulheres na indústria brasileira. Revista Brasileira de Economia, Rio de Janeiro., v. 37, n. 4, p. 435-480, out/dez 1983.

CARRERA-FERNANDEZ, J.; MENEZES, W. F. Discriminação interna aos mercados formal e informal de trabalho da região metropolitana de Salvador. Anais do XXVI Encontro Nacional de Economia - ANPEC, Vitória - ES, dezembro 1998. 
CAVALIERI, C. H.; FERNANDES, R. Diferenciais de salários por gênero e cor: uma comparação entre as regiões metropolitanas brasileiras. Revista de Economia Política, São Paulo, v. 18, n. 1 (69), p. 158-175, janeiro-março 1998.

FERNANDES, R.; DE FELÍCIO, F. Married women's labor force participation as a response to the husband's unemployment in Brazil. Anais do XXIV Encontro Nacional de Econometria - SBE, Nova Friburgo - RJ, dezembro, 2002.

IKEDA, M. "Segregação por gênero" no mercado formal de trabalho. Revista do BNDES, Rio de Janeiro, v. 7, n. 13, p. 89-106, junho 2000.

JANSEN, S. L. Análise econométrica das diferenças salariais por gênero no mercado de trabalho brasileiro. Porto Alegre, UFRGS, 1999. (Monografia).

JATOBÁ, J. Oferta de força de trabalho familiar e crise econômica: Brasil metropolitano, 1983. Revista de Econometria, Rio de Janeiro, v. X, n. 2, p.195-223, 1990.

LOUREIRO, P. R. A. Uma resenha teórica e empírica sobre a economia da discriminação. Revista Brasileira de Economia, Rio de Janeiro, v. 57, n. 1, p. 125-157, janeiro-março 2003.

MACEDO, R. Diferenciais de salários entre empresas privadas e estatais no Brasil. Revista Brasileira de Economia, Rio de Janeiro, v. 39, n. 4, p. 437-448, outubro-dezembro 1985.

MADDALA, G.S. Introduction to econometrics. Second edition. Prentice Hall, New York. 1992.

MADDEN, J. F. Discrimination - a manifestation of male market power? In: Sex, discrimination and the division of labor, Columbia University Press, New York, chap. 6, p. 146-174, 1975.

OMETTO, A. M. H. Participação da mulher no mercado de trabalho: segregação e discriminação em Pernambuco e São Paulo. Piracicaba, ESALQ/USP, 1997. (Tese de doutorado)

OMETTO, A. M. H.; HOFFMANN, R.; ALVES, M. C. Participação da mulher no mercado de trabalho: discriminação em Pernambuco e São 
Paulo. Revista Brasileira de Economia, Rio de Janeiro, v.53, n.3, p. 287-322, 1999.

OAXACA, R. Male-female wage differentials in urban labor markets. International Economic Review, v. 62 , p. 693-709, 1973.

OAXACA, R. Economics of discrimination. The New Palgrave: a dictionary of economics. London, MaccMillan, pg. 3756-3762, 1987.

OAXACA, R.; RANSON, M. R. Identification in detailed wage decompositions. The Review of Economics and Statistics. V. 81, p. 154-157, 1999.

PHELPS, E. S. The statistical theory of racism and sexism. American Economic Review, LXII, p. 659-661. 1972.

RAMOS, L.; SOARES, A. L. Participação da mulher na força de trabalho e pobreza no Brasil. Revista de Economia Política, São Paulo, v. 15, n. 3 (59), julho-setembro, 1995.

REIS, J. G. A.; BARROS, R. P. Desigualdade salarial: resultados de pesquisas recentes. Distribuição de Renda no Brasil, São Paulo, Paz e Terra, 1991.

ROBINSON, J. The economics of imperfect competition. Macmillan, London, 1933.

SACCONATO, A. L.; MENEZES-FILHO, N. A. A diferença salarial entre os trabalhadores americanos e brasileiros: uma análise com micro dados. Anais do XXVIII Encontro Nacional de Economia - ANPEC, Campinas, dezembro, 2000.

SANCHES, S. A Outra Realidade do Mercado Brasileiro. Jornal dos Economistas, Rio de Janeiro, n. 131, p.8-10, 2000.

SCORZAFAVE, L. G.; MENEZES-FILHO, N. A. A evolução da participação feminina no mercado de trabalho brasileiro. Anais do XXVIII Encontro Nacional de Economia - ANPEC, Campinas - SP, dezembro 2000.

SPENCE, M. Job market signaling. Quarterly Journal of Economics, LXXXVII, p. 355-374, 1973. 


\section{Apêndice}

Tabela 7 - Distribuição dos trabalhadores segundo gênero e nível de escolaridade

\begin{tabular}{lccc}
\hline \multicolumn{1}{c}{ Escolaridade } & Homem (\%) & Mulher (\%) & Total (\%) \\
\hline Sem instrução & 1,05 & 1,31 & 1,16 \\
$1^{\circ}$. grau incompleto & 74,28 & 71,74 & 73,23 \\
$1^{\circ}$. grau completo & 12,58 & 12,44 & 12,53 \\
$2^{\circ}$. grau incompleto & 5,80 & 6,32 & 6,01 \\
$2^{\circ}$. grau completo & 6,06 & 7,88 & 6,80 \\
Superior incompleto & 0,15 & 0,23 & 0,19 \\
Superior completo & 0,08 & 0,08 & 0,08 \\
Total & 100,00 & 100,00 & 100,00 \\
\hline
\end{tabular}

Fonte: Cálculos do autor.

Tabela 8 - Média amostral para salários, idade, escolaridade e tempo de emprego - trabalhadores homogêneos quanto à ocupação e escolaridade ( $1^{\circ}$ grau de escolaridade incompleto)

\begin{tabular}{lccc}
\hline \multicolumn{1}{c}{ Variáveis } & Homem & Mulher & Total \\
\hline Salários & 2,6829 & 2,5911 & 2,6453 \\
Idade & 25,9298 & 27,3123 & 26,4967 \\
Até 2,9 meses & 0,1337 & 0,1009 & 0,1203 \\
3 a 5,9 meses & 0,0822 & 0,0410 & 0,0653 \\
6 a 11,9 meses & 0,1776 & 0,1498 & 0,1662 \\
12 a 23,9 meses & 0,1732 & 0,1261 & 0,1539 \\
24 a 35,9 meses & 0,1206 & 0,1119 & 0,1170 \\
36 a 59,9 meses & 0,1403 & 0,2066 & 0,1675 \\
60 a 119,6 meses & 0,1151 & 0,2034 & 0,1513 \\
120 ou mais meses & 0,0570 & 0,5990 & 0,0582 \\
\hline
\end{tabular}

Fonte: Cálculos do autor. 
Tabela 9 - Resultados da equação de salários para homens e mulheres Trabalhadores homogêneos quanto à ocupação e escolaridade ( $1^{\circ}$ grau de escolaridade incompleto)

\begin{tabular}{|c|c|c|c|c|}
\hline \multirow{2}{*}{ Variável } & \multicolumn{2}{|c|}{ Homens } & \multicolumn{2}{|c|}{ Mulheres } \\
\hline & Coeficientes & $\mathrm{t}$ & Coeficientes & $\mathrm{t}$ \\
\hline Constante & 0,3923 & $(0,2545)$ & 0,8324 & $(0,1182)$ \\
\hline Idade & 0,0197 & $(0,0194)$ & $-0,0154$ & $(0,0082)$ \\
\hline Idade2 & $-0,0358$ & $(0,0344)$ & 0,0206 & $(0,0132)$ \\
\hline 3 a 5,9 meses & 0,1131 & $(0,0234)^{\mathrm{c}}$ & 0,1435 & $(0,0485)^{\mathrm{C}}$ \\
\hline 6 a 11,9 meses & 0,1209 & $(0,0193)^{\mathrm{C}}$ & 0,1834 & $(0,0339)^{\mathrm{C}}$ \\
\hline 12 a 23,9 meses & 0,2726 & $(0,0201)^{\mathrm{C}}$ & 0,2422 & $(0,0355)^{\mathrm{C}}$ \\
\hline 24 a 35,9 meses & 0,3337 & $(0,0274)^{\mathrm{C}}$ & 0,3510 & $(0,0374)^{\mathrm{c}}$ \\
\hline 36 a 59,9 meses & 0,3870 & $(0,0269)^{\mathrm{c}}$ & 0,4226 & $(0,0337)^{\mathrm{C}}$ \\
\hline 60 a 119,6 meses & 0,5562 & $(0,0290)^{\mathrm{C}}$ & 0,5427 & $(0,0341)^{\mathrm{C}}$ \\
\hline 120 ou mais meses & 0,8819 & $(0,0609)^{\mathrm{C}}$ & 0,6969 & $(0,0452)^{\mathrm{c}}$ \\
\hline $\mathrm{R}^{2}$ & 0,52 & & 0,41 & \\
\hline Teste F & 148,27 & & 51,64 & \\
\hline $\mathrm{N}$ & 912 & & 634 & \\
\hline SQR & 40,29 & & 27,06 & \\
\hline
\end{tabular}

Fonte: Cálculos do autor

Obs.: (a) significante a 10\%, (b) significante a $5 \%$ e (c) significante a $1 \%$. Os valores entre parêntesis referem-se aos desvios padrões robustos à heterocedasticidade.

Recebido em outubro de 2004 e revisto em julho de 2005 